\title{
Robust Realism for the Life Sciences
}

\author{
Markus I. Eronen
}

markus.eronen@kuleuven.be

forthcoming in Synthese

\begin{abstract}
Although scientific realism is the default position in the life sciences, philosophical accounts of realism are geared towards physics and run into trouble when applied to fields such as biology or neuroscience. In this paper, I formulate a new robustness-based version of entity realism, and show that it provides a plausible account of realism for the life sciences that is also continuous with scientific practice. It is based on the idea that if there are several independent ways of measuring, detecting or deriving something, then we are justified in believing that it is real. I also consider several possible objections to robustness-based entity realism, discuss its relationship to ontic structural realism, and show how it has the potential to provide a novel response to the pessimistic induction argument.
\end{abstract}

Keywords: scientific realism; life sciences; robustness; entity realism 


\section{Introduction}

The basic idea of scientific realism is that the best scientific theories are approximately true and the entities they refer to are real. Scientific realism is probably even more widespread in the life sciences than in physics: Whereas many theoretical physicists take an antirealist stance towards fundamental theories and entities (see, e.g., Zeilinger 2005), it is hard to find a biologist who doubts the reality of micro-organisms or DNA molecules. However, philosophical accounts of scientific realism tend to run into problems when applied to the life sciences. This is in particular true for the currently popular ontic structural realism, according to which all there exists is structure, and continuity in scientific change should be understood as continuity at the level of structure (Frigg and Votsis 2011; Ladyman 2014). For example, Steven French' (2011) discussion of scientific realism in biology merely points towards possible ways in which structural realism could be extended to biology in future work. James Ladyman and Don Ross are more ambitious, and apply their ontic structural realism to the special sciences, but at the cost of reducing all special science entities to patterns that are defined in highly technical information-theoretic terms (Ladyman and Ross 2007). This makes their realism completely detached from scientific practice, providing no tools for developing or understanding science-based arguments for the reality of special science entities and properties.

In contrast, entity realism as formulated by Cartwright (1983) and Hacking (1983) is based on the actual scientific practices of experimental manipulation and causal explanation. Its core idea is that even though we may not be warranted in believing in the truth of scientific 
theories, we are warranted in believing that the entities that scientists causally manipulate are real. In this way, entity realism (ER) seems to constitute a form or realism that is plausible from the point of view of everyday science, and easily applicable to the life sciences.

However, in discussions of scientific realism, ER is often quickly dismissed (see, e.g., Devitt 2005; Ladyman and Ross, 2007; Psillos 1999), and it has few proponents nowadays. This is probably due to the several strong counterarguments that have been raised against it. In this paper, I will formulate a new version of ER, and based on an example from science, show that this version has resources to answer all the objections raised against original ER. What makes this account novel and attractive in comparison to original ER and other forms of realism inspired by it (Chakravartty 2007; Egg 2012, 2016; Suarez 2008 ) is that it moves beyond causal motivations for realism, and is based on the notion of robustness instead. Roughly, the idea is that if there are many independent ways of measuring or detecting an entity, we are highly justified in believing that the entity is real.

Robustness reasoning of this kind also briefly appears in the context of original ER. However, Cartwright (1983, p. 84) mentions it only to supplement her "inference to the probable cause" strategy, and does not develop it into an independent argument for realism. Hacking (1981) discusses robustness (or "the argument from coincidence") in the context of seeing through microscopes, but somewhat surprisingly claims that this has little relevance for debates on scientific realism. In contrast, I will argue that robustness, when properly understood, in fact leads to a strong argument for scientific (entity) realism. I will 
also show that, in contrast to ontic structural realism, robustness-based ER helps understand how ontological commitments arise from scientific practice, and is perfectly suited for capturing the kind of commonsense scientific realism that is evident in the life sciences. Moreover, robustness-based ER leads to a novel way of responding to pessimistic induction arguments against scientific realism.

In the next section, I will briefly go through ER and its main problems. In Section 3, I will present the robustness argument for ER, and in Section 4, I will elaborate on robustnessbased ER and possible objections based on an example from science (amacrine cells). In Section 5, I consider robustness-based ER as an account of scientific realism in the life sciences, and its relationship to (ontic) structural realism.

\section{Entity Realism}

The classic accounts of ER are in Nancy Cartwright's (1983) How the Laws of Physics Lie and Ian Hacking's (1983) Representing and Intervening. The most important argument for ER, at least for Cartwright, is based on the supposedly special nature of causal explanation. Cartwright (1983) argues extensively that accepting a causal explanation implies belief in the causes described by the explanation. In other words, "to accept the explanation is to admit the cause" (ibid., 99), or "inference from effect to cause is legitimate" (ibid., 89). To illustrate, she gives the following example (ibid., 91). The lemon tree in her garden is sick, and the leaves are falling off. She comes up with an explanation: Water has accumulated at the bottom of the pot, which has made the tree sick. According to Cartwright, accepting 
this explanation as correct requires believing that the cause is real; it is not possible to believe that the explanation is correct without believing that there really is water at the bottom of the pot. ${ }^{1}$

Cartwright (1983) and Hacking (1983) also appeal to scientific practice and experimentation for support. Hacking famously argues that the best evidence for the reality of electrons is that we can use them to create and study other phenomena - "if you can spray them, they are real" (Hacking 1983, p. 23). He also claims that practicing scientists cannot help being realists about (experimental) theoretical entities (Hacking 1983, p. 262). Cartwright argues that experimentation can give causal explanations a degree of objectivity that is impossible to reach for laws and theories, referring for example to a laser-making company that runs numerous test lasers to death each year to make sure that the lasers produced have exactly the effects that they are supposed to have (Cartwright 1983, p. 3). Finally, an important consideration in favor of ER is that entities seem to be more stable and resistant to scientific revolutions than theories and laws. For example, the electron entered the ontology of physics in the late $19^{\text {th }}$ century, and has remained there ever since, although theories in physics have gone through such dramatic changes as the discovery of quantum mechanics and the relativity theory (Hacking 1982). Thus, ER appears to be less susceptible to the pessimistic induction argument (i.e., the argument against realism based

\footnotetext{
${ }^{1}$ The exact structure of Cartwright's original argument is not clear. For an overview of different interpretations and their problems, see Hitchcock (1992).
} 
on the observation that many successful theories of the past have turned out to be false; Laudan 1981) than standard scientific realism.

ER is an attractive position, as it seems to amount to "defensible middle ground" between antirealism and standard scientific realism (Clarke 2001): Entity realists accept the antirealist tenet that we are not required to believe in the truth of scientific theories, but also defend limited realism, as they argue that we are warranted in believing that some entities that appear in scientific explanations are real. However, ER faces serious counterarguments that have made the position unpopular.

First of all, the notion that accepting a causal explanation implies belief in the reality of the cause is highly questionable. To see this, note that there is an important difference between accepting a causal explanation and believing that a causal explanation is true (Hitchcock 1992). An antirealist along the lines of van Fraassen (1980) can argue that whereas the latter does imply believing that the cause is real, the former does not. When scientists accept a causal explanation, they can just believe that there is a causal story that is empirically adequate (Hitchcock 1992). This antirealist approach to causal explanation also seems to be supported by the history of science: The caloric fluid appeared in widely accepted causal generalizations in the $19^{\text {th }}$ century, but we now know that it doesn't exist (Hitchcock 1992; Radder 2012, 109). The connection between causal explanation and scientific realism remains a controversial issue and also defenses of causation-based arguments for realism are found in the literature (Egg 2012, 2016; Suarez 2008), but what 
is clear is that Cartwright's argument in its original form is not compelling (see also Clarke (2001) and Hitchcock (1992) for more discussion). ${ }^{2}$

A second problem for ER is that it seems to lead back to a form of standard scientific realism (Morrison 1990; Psillos 1999, p. 249). An entity realist cannot just believe that some brute entity $\mathrm{X}$ exists without believing anything else about that entity. At the very least, she has to accept the reality of some key properties of the entity, for example, that the electron has a negative electric charge. However, the only scientifically acceptable way to attribute properties to entities is to do this on the basis of the most state-of-the-art scientific theories. This, in turn, seems to require believing that those theories are to some extent true (Chakravartty 2007, p. 30; Psillos 1999, pp. 248-249), and accepting that the best scientific theories are to some extent true arguably amounts to accepting standard scientific realism. In this way, entity realism seems to collapse to standard scientific realism.

A third and related problem is that, in spite of appearance, ER may not fare any better than the alternatives against the argument from pessimistic induction (Morrison 1990; Chakravartty 2007, p. 32). If the reasoning in the previous paragraph holds, and properties are attributed to entities based on the best available scientific theories, then scientific change also results in changes in the properties attributed. This seems to be supported by history of science: Whereas the electron as an entity has withstood several scientific

\footnotetext{
${ }^{2}$ Suarez (2008) and Egg $(2012,2016)$ defend entity realism by arguing that, when the right conditions are satisfied, causal explanation does give warrant for believing that the cause is real. This strategy is interesting, but I will not discuss it in detail here, as it leads to a form of entity realism that is not well suited for accounting for scientific realism in the life sciences - see section 4 for more.
} 
revolutions, views about its properties have considerably changed. Thomson and Rutherford believed very different things about electrons than scientists do today (Morrison 1990; Chakravartty 2007, p. 32) - for example, they did not believe that the electron is a fermion or that it exhibits wave-particle duality, which are nowadays seen as fundamental properties of electrons. Although entity realists can appeal to the causal theory of reference to argue that contemporary scientists are still talking about the same electron as Thomson and Rutherford (e.g., Hacking 1982), this continuity is very shallow if the views about the nature and the properties of the electron have dramatically changed in the meantime.

In sum, the main problems of ER are that (1) it is not clear why the success of causal explanation would warrant inferring the reality of the cause; (2) belief in the reality of the properties of entities seems to imply accepting that the best scientific theories are to some extent true, leading to standard scientific realism; and (3) the solution to the pessimistic induction that ER provides is too shallow to be interesting, as it does not apply to the properties of entities. In the next section, I will formulate a new robustness-based version of ER, and in Section 4, I will show how it can be used to successfully tackle these problems.

\section{Robustness-Based Entity Realism}

As we saw above, ER in the form defended by Cartwright and Hacking is deeply problematic, and therefore does not help in justifying or understanding the rampant 
commonsense realism in the life sciences. In order to overcome these problems, I will now introduce a novel version of ER, building on the notion of robustness. Roughly, something is robust if there are several independent ways of measuring, detecting, producing or deriving it. ${ }^{3}$ More specifically, the idea is that if there are many ways of measuring, detecting, producing or deriving something, and those ways are sufficiently independent, then it is very unlikely that all of them turn out to be mistaken or erroneous (Chang 2004; Egg 2016, Kuorikoski and Marchionni 2016; Schupbach forthcoming; Soler, Trizio, Nickles, and Wimsatt 2012; Wimsatt 1981, 1994, 2007). Thus, things that are robust in this sense are very likely to be real (Wimsatt 1981, 2007). ${ }^{4}$ For example, neurons can be measured, detected and even produced with many different techniques and setups relying on different theoretical assumptions. Consequently, they are robust and extremely likely to be real.

\footnotetext{
${ }^{3}$ This idea has also many other names, including triangulation, overdetermination, mutual grounding, diverse testing, argument from coincidence, and so on. Note also that this use of the term 'robustness' should not be confused with the distantly related notion of 'robustness analysis' that has been much discussed in the context of modeling (e.g., Weisberg 2006; Odenbaugh and Alexandrova 2011; Kuorikoski, Lehtinen and Marchionni 2012). See Calcott (2011) and Eronen (2015) for more on the differences between various notions of robustness.

${ }^{4}$ This can also be seen as a common cause argument: The independent pieces of evidence are explained by being due to a common cause, i.e., the robust entity or property (see, e.g., Salmon 1984). However, the account of robustness I offer below is more general and includes dimensions (e.g., derivability and explanatory role) that do not easily fit into the common cause framework.
} 
Robustness is sometimes put forward as an ontological criterion for what is real (cf. Wimsatt 1994, 2007), but this seems too strong, as robustness is rather a fallible, relative and epistemic notion. Instead, the connection between robustness and reality should be understood as follows (Eronen 2012, 2015): Robustness confers justification for believing that $X$ is real, and the degree of this justification corresponds to the degree that we have robust evidence for $X$. In this way, robustness is seen as a source of justification for ontological commitments, and not as an ontological criterion. An advantage of this approach is that it makes robustness a sufficient but not necessary condition for ontological justification, and thus leaves open the possibility that there may be also other sources of such justification (Eronen 2015). Consequently, cases where we are apparently warranted in believing in the reality of something that is not robust (e.g., states of phenomenal consciousness) are not counterarguments to this account (ibid.).

Robustness comes in degrees and is relative to a certain scientific community at a certain time - for example, there was no robust evidence for DNA molecules in the $19^{\text {th }}$ century, but now that evidence is abundant. This needs to be incorporated into the definition of robustness, which can be done as follows (Eronen 2015):

(Robustness) The relevant scientific community at a certain time has robust evidence for $\mathrm{X}$ insofar as $\mathrm{X}$ is detectable, measurable, derivable, producible or explanatory in a variety of independent ways.

The notion "explanatory" in this definition requires some clarification. It has been included for the reason that it is very plausible that things that appear in many independent 
explanatory generalizations or models are more robust $t^{5}$ than things that do not (ibid.). For example, one factor that contributes to the robustness of entities such as electrons or action potentials is that we have several good models and generalizations that describe and explain their behavior. The definition also implies that none of the dimensions (detectable, measurable, derivable, etc.) is by itself necessary for robustness. The moon, for example, is an extremely robust entity, although there is no clear sense in which we can produce it, and entities or properties can be highly robust even though there are no accepted explanatory generalizations or models involving them (e.g., gamma-ray bursts).

The notion of independence is crucial for robustness: If different ways of measuring something are not independent from each other, but are based on the same assumptions and methods, then they all lose their value if those assumptions and methods turn out to be false or mistaken, and the robustness that they confer is only illusory. This independence can be roughly characterized as follows: Ways of measuring, modeling or detecting are maximally independent when they are based on different kinds of causal mechanisms and theoretical assumptions (cf. Kuorikoski and Marchionni 2016). This ensures that when one way of measuring, modeling or detecting turns out to be faulty, it does not undermine the validity of the others. ${ }^{6}$ For example, Golgi's method to detect neurons is based on impregnating nervous tissue with chemicals, which then results in a few neurons in the

\footnotetext{
${ }^{5}$ Strictly speaking, it would be more accurate to always write "robust evidence for $\mathrm{X}$ " instead of " $\mathrm{X}$ is robust", but for the sake of readability, I also use the latter kinds of expressions here.

${ }^{6}$ For more discussion on robustness and independence, see Hudson (2014), Kuorikoski and Marchionni (2016), Schupbach (forthcoming) and Stegenga (2009).
} 
tissue becoming black and thus easily discernible with a regular light microscope (Lichtman, Livet and Sanes 2008). Electron microscope imaging of neurons is based on firing a beam electrons through a thin section of nervous tissue, which results in part of the electrons scattering due to interaction with the specimen, and thus the beam that passes through the sample contains information about the shape and structure of the specimen that can then be visualized as a photograph or on a computer screen (Briggman and Bock 2012). Golgi's method and electron microscope imaging are therefore based on different kinds of causal mechanisms and different theoretical assumptions, and independent in the right way.

Based on the above, we can formulate a rather straightforward argument for ER. First, it is clear that many entities in science are detectable, measurable, derivable, producible, or explanatory in a variety of independent ways, and thus we have a high degree of robust evidence for them. Second, it follows from this that we have a high degree of justification in believing that many entities in science are real. Third, if we have a high degree of justification for believing in the reality of many entities in science, then we have a high degree of justification for believing that ER is true. I will now proceed to clarify this robustness-based entity realism (from now on RER) further based on a scientific example, and respond to possible objections.

\section{Amacrine Cells and Objections}


The main example that I will use here to elaborate on RER is amacrine cells in the retina. Amacrine cells are found in the layer of the retina (inner plexiform layer) where bipolar cells, transmitting signals from the photoreceptors (rods and cones), connect with ganglion cells, which then convey the visual information further into the brain (Kolb 2015). Amacrine cells come in many subtypes, and carry out a broad range of functions, for example integrating signals from bipolar cells and modulating the activity of ganglion cells and other types of retinal cells (Masland 2012).

This case is well-suited for the present purposes, as amacrine cells are "theoretical" entities in the sense that they are not directly observable, and their evidential status has gone through a dramatic evolution. In the late $19^{\text {th }}$ century, when Ramón y Cajal described, visualized and named amacrine cells, there were only a few ways of detecting them, most importantly Golgi's method (Guillery 2005; Kolb 2015). However, nowadays there is overwhelmingly robust evidence for them. To mention just some examples: amacrine cells can be observed with a broad range of staining techniques; they can be seen with high power light microscopes and imaged with electron microscopes; their activity can be recorded with various single-cell and multi-unit recording setups; they play an important role in explanatory models and generalizations concerning retinal processing, and so on (Kolb 2003). Even if broad categories of these sources of evidence would turn out to be mistaken, plenty of other independent sources would still remain, and we would still have highly robust evidence for amacrine cells. 
With this example in mind, let us go through possible objections to RER, starting from the objections outlined in Section 2. The first problem was that, contra Cartwright (1983), accepting a causal explanation does not seem to imply accepting the reality of the cause. This problem does not threaten RER, which does not appeal to any special features of causal explanation, in contrast to the original ER (and later versions such as Egg (2012, 2016) and Suarez (2008)). In RER, the fact that an entity or a property appears in a causal generalization can contribute to its robustness, but just as one possible factor among many others. A robustness-realist can perfectly well accept that having a successful causal explanation does not alone provide justification for believing in the reality of the cause.

However, the antirealist can reformulate the objection so that it applies to robustness. A constructive empiricist in the vein of van Fraassen can insist that there is no compelling reason why anyone would be required (as opposed to permitted) to believe in entities for which we have robust evidence (see also van Fraassen 1985, pp. 297-300). It is true that, strictly speaking, no one is required to believe in the reality of robust entities. However, while in the case of causal explanation the suspension of belief in the reality of the cause is defensible and perhaps even prudent, in the case of highly robust entities like amacrine cells it borders on irrationality. Can someone who has access to all the robust evidence for amacrine cells rationally refuse to believe in their reality? Perhaps, but in roughly the same sense that she can rationally refuse to believe in the reality of palm trees (see also Hacking 
1981). ${ }^{7}$ The antirealist position comes dangerously close to outright skepticism in the case of highly robust entities.

The second problem for ER was that, in order to be interesting, ER needs to be extended to properties of entities, and this seems to imply that the best scientific theories are to some extent true, leading to standard scientific realism. First of all, RER indeed applies to properties as well, and not just the entities themselves. The electrical conductivity of iron is detectable, measurable, derivable, producible and explanatory in a broad range of independent ways, and is thus an extremely robust property. Having a membrane potential is an extremely robust property of the neuron, transmitting action potentials is an extremely robust property of the axon, and so on. However, extending RER to properties has only minimal implications for the truth of theories. The requirement of independence guarantees that the robust evidence for an entity or property does not rely on just one model or theory, but on many distinct models or theories. Any one of these models or theories could turn out to be false, and the entity or property would still remain robust. For example, even if a specific computational model of retinal processing involving amacrine cells turns out to be false, plenty of other sources of independent evidence for amacrine cells (e.g., the various staining techniques) will still remain. Moreover, many ways of detecting or measuring the properties of amacrine cells do not depend on any theory, except perhaps some very

\footnotetext{
${ }^{7}$ However, many other aspects of the constructive empiricism of van Fraassen (1980) are in fact compatible with RER: For example, RER does not require accepting inference to the best explanation as valid for theories and explanations.
} 
shallow descriptive theory. ${ }^{8}$ For example, Golgi's staining method was developed over 130 years ago and is still in use, but there is no accepted theory that would explain how it actually works (Guillery 2005, p. 1290).

One might object that in order to be sure that the different independent ways are measuring the same thing, we need some overarching theory that connects them. However, this need not be the case. If several measurement techniques consistently and repeatedly pick up the same pattern independently of each other (e,g., the dendritic spines of amacrine cells), this alone is sufficient evidence that they are measuring the same thing. In any case, the claim here is not that in RER one does not have to believe in any (parts of) theories at all; the point is rather that the theoretical commitments can remain very minimal. For example, the robustness realist may be required to believe in shallow descriptive theories concerning the robust entities and their properties, and that there are at least some true elements among the various theories and models involved. However, she can still remain entirely agnostic about the truth of (explanatory or fundamental) scientific theories more generally, and deny the reliability of arguments based on inference the best explanation (IBE), i.e., arguments to the effect that the best explanation for the success of scientific theories is that they are approximately true. Perhaps these minimal theoretical commitments that RER requires are enough to make some philosophers treat it as a form of standard scientific realism, but even if this is the case, it does not as such affect the plausibility of RER. If

\footnotetext{
${ }^{8}$ The extent to which measuring a property (or believing in the reality of a property) requires belief in theories also depends on how broadly we understand 'theory'. For more on the relationships between properties, theories and realism, see Chakravartty (2007, ch. 2-3).
} 
RER is a form of standard scientific realism, it is a very specific form, and one that is resistant to antirealist counterarguments such as the pessimistic induction argument (see below).

There is, however, a related concern that can be raised regarding the novelty of RER: What if robustness reasoning is just IBE in disguise? RER could be construed as claiming that the best explanation for the robust evidence for $\mathrm{X}$ is that $\mathrm{X}$ is real, so we are justified in believing that $\mathrm{X}$ is real (cf. Hudson 2014). Similarly, the argument for RER could be rendered as a common cause IBE: The best explanation for different kinds of independent evidence is that they are due to a common cause (X). Either way, RER seems to be relying on the same pattern of reasoning as standard scientific realism. However, it is perfectly possible to argue that a certain specific kind of IBE is reliable, without accepting that other instances of IBE are reliable (Clarke 2001). A robustness-realist can defend the view that we have very good reasons to rely on the robustness-based IBE that concludes that we are highly justified in believing that $\mathrm{X}$ is real, while also accepting that the IBE from the success of theories to their truth is very problematic. Thus, the mere fact that RER can be construed as relying on IBE does not in any way undermine it.

The third problem for ER was that the views about the key properties of entities tend to change as science progresses, rendering the continuity in entity realism shallow and uninteresting. However, this issue can be reformulated and examined in new light once we understand that we can also have varying degrees of robust evidence for properties. Arguably, highly robust properties tend to be resistant to scientific change (see also 
Chakravartty 2007, ch. 2). Properties in biology for which there was highly robust evidence in the early $20^{\text {th }}$ century, such as the negative transmembrane potential of neurons, have been retained (Guillery 2005). Although antirealists have presented several cases of entities or properties that were eliminated, these do not include entities or properties that were robust to a very high degree.

This reasoning can be further developed into a full-blown response to pessimistic induction. Throughout the modern scientific era, new scientific methods and measurement instruments have been developed at an increasing rate, and in general, growth of science and technology does not seem to be linear, but exponential (Bornmann and Mutz 2015). This is also clearly seen in the example of the amacrine cells: the number of different ways of detecting and measuring amacrine cells and their properties has not only increased, but is now of a completely different order than 100 years ago. Thus, the evidence that we currently have for scientific entities such as amacrine cells (and their properties) is vastly more robust than the evidence for any entities or properties in the history of science that were subsequently eliminated (such as the caloric). This is a serious problem for antirealist arguments that rely on inductions over cases from the history of science: If there is a jump in the degree of robust evidence for current entities and properties when compared to the historical entities and properties that were eliminated, then pessimistic induction against entity realism does not get off ground. ${ }^{9}$

\footnotetext{
${ }^{9}$ See Fahrbach (2011) for a different but complementary argument against pessimistic induction based on the exponential growth of science.
} 
I have focused here on the case of amacrine cells, but this is not an isolated or cherrypicked example. The life sciences are full of entities and their properties for which we have similarly robust evidence: mitochondria, cell membranes, pollen, the Eschericia coli bacterium, and so on. Importantly, the fact that amacrine cells can be observed with the help of microscopes and imaged with various staining techniques plays no special role here, as also properties that cannot be similarly visualized can be very robust - consider, for example, the membrane potential of cells, body temperature, or blood glucose level. In sum, none of the objections raised against ER undermine the plausibility of RER. It is a viable and defensible form of scientific realism that should to be taken seriously and explored in more detail.

\section{Robust Realism for the Life Sciences ${ }^{10}$}

As I pointed out in the introduction, an important drawback of the currently popular alternative form of limited realism, ontic structural realism (OSR; see Ladyman and Ross 2007; Ladyman 2014), is that it is detached from scientific practice outside physics. Its core idea is that reality is in a fundamental sense relational or structural in nature (O’Conaill 2014). It provides a metaphysical account of what exists (i.e, structure) and what continuity in scientific change amounts to (i.e., the retention of structure). One of the main motivations for OSR is that the state-of-the-art theories in fundamental physics

\footnotetext{
${ }^{10}$ Much of this section was written in response to insightful comments by two anonymous reviewers, for which I am very grateful.
} 
postulate only relations and structures, and not objects and properties in the everyday or traditional metaphysical sense (Ladyman 2014). However, in special sciences such as biology, objects (e.g. organisms, cells and species) and their properties play a crucial role, and therefore it is not surprising that OSR runs into problems when applied to the special sciences (Frigg and Votsis 2011; Lyre 2013). ${ }^{11}$

In contrast to OSR, RER supports realism in the life sciences in a way that is continuous with scientific practice and the ontological commitments of scientists. It explains how scientific practice results in robust evidence and thereby in justification for believing that entities and properties, as defined and studied by scientists, are real. Moreover, as the examples in the previous section show, continuity in the life sciences can very well be understood in terms of retention of entities and properties, while it is far from clear to what extent it could be accounted for in terms of retention of structure. There is extremely (and increasingly) robust evidence for a broad range of entities and properties in the life sciences, such cell membranes or pollen, and they have been retained in the ontology of biology in spite of scientific revolutions such as the discovery of the DNA.

\footnotetext{
${ }^{11}$ What this means for OSR is an open question that goes beyond the scope of this paper. It is possible, for example, that the ontological commitments that RER supports could somehow be translated to the objectless ontology of OSR (cf. Ladyman and Ross 2007), rendering the two compatible. On the other hand, it has also been argued that there is no need to expand OSR to the special sciences (Lyre 2013). Note also that even if it is true that there are no objects but only structure in the ontology of fundamental physics, this is not a threat to RER, as RER does not imply or require that only entities or properties exist, or that robustness is the only route to ontological commitments and justification (see section 3 ).
} 
One might object that the same reasoning can be applied to standard scientific realism in the life sciences: Many of the central theories in biology, such as the theory of evolution by natural selection or the fundamental principles of cell biology (see, e.g., Fagan 2013), have also been retained and are extremely robust. However, as already suggested in section 4 , there is no reason why a supporter RER would have to insist that standard scientific realism is completely mistaken. It may well be true that robustness reasoning can also be applied to theories such as the theory of natural selection, but this does not mean that RER is false, or "collapses to" or is "just a form of" standard scientific realism. RER differs fundamentally from standard scientific realism by relying on the robustness argument instead of the explanatory and predictive success of theories, and can be applied also when there are no accepted theories available, which is not unusual in the life sciences. Thus, if it turns out that RER applies not only to entities and properties but also to some biological theories, this does not refute the account, but simply expands its scope.

It is also important to note that I am not assuming that life scientists would in general identify themselves as entity realists rather than ontic structural realists or standard scientific realists. It is likely that most practicing scientists are not even aware of these distinctions. The point is rather that OSR and standard scientific realism are insufficient for understanding realism in the life sciences, and RER fills this gap by providing a conceptual account of the ontological commitments implicit in the scientific practice in the life sciences. 
RER also has important advantages to other recently proposed forms of entity realism. Suarez $(2008)$ and Egg $(2012,2016)$ have provided responses to the arguments against ER outlined in section 2, and argue that in the right conditions (e.g., when the explanation is non-redundant and there is a well-defined notion of what it means to modify the target property) causal explanation does provide warrant for believing that the cause is real. Like OSR, this approach runs into difficulties when applied to realism in the life sciences. Biological researchers are often highly justified in believing in the reality of entities or properties even though there is no causal warrant for them in the sense of having a clear idea of their causal role or how to modify them: This was the case, for example, for the conduction velocities of action potentials or the dendritic spines of amacrine cells in late $19^{\text {th }}$ century. Moreover, the causal warrant approach does not provide an account of the variation in degrees of confidence in the reality of entities or properties in science. In particular, it does not provide an explanation for the extremely high confidence in the reality of entities such as amacrine cells or their properties, which is an important resource for responding to the pessimistic induction argument (see section 4), and is very naturally accounted for by RER.

Finally, an interesting feature of RER is that it has the implication that we are often more justified in believing in the reality of biological entities and properties than fundamental physical entities or properties (see also Eronen 2015). For example, when compared to the neuron, there are relatively few independent ways of measuring, detecting or producing the up quark. Same applies to the recently detected Higgs boson, and to various other elementary particles. However, this does not mean that biological entities or properties are 
more real or more fundamental than physical ones, but simply that we sometimes have more robust evidence for them.

\section{Concluding Remarks}

Entity realism has gone out of fashion in philosophy of science, mainly due to serious arguments presented against it, and the rise of attractive alternatives such as OSR.

However, I have argued in this paper that RER can deal with all the counterarguments raised against entity realism, and offers a more credible account of realism for the life sciences than OSR. It also opens up the possibility for developing a novel solution to the pessimistic induction argument.

Although I have focused here on the life sciences, RER could potentially be expanded to other special sciences. Some disciplines bring along interesting new challenges, for example psychology, where it is not clear to what degree there is robust evidence for key constructs such as the $g$ factor of intelligence. It is also important to reemphasize that in RER robustness is seen as a sufficient but not necessary condition for ontological justification, so if a discipline has its own proprietary means of justifying ontological commitments, RER does not need to be in conflict with them.

Due to its connection with scientific practice, RER also has the potential to shed light on the ontological commitments of scientists, and to contribute to practice-oriented accounts of scientific progress, reductionism, unification and other issues. However, these are topics 
for future research - the main aim of this paper has been to show that robustness-based entity realism is a plausible account of scientific realism in the life sciences.

\section{Acknowledgments}

I thank Laura Bringmann, Daniel Brooks and Leah Henderson for very helpful critical comments on earlier drafts of the paper. I am also grateful to two anonymous referees, and the audiences at Philosophy of Science in a Forest 2016 (Utrecht), PSA 2016 (Atlanta) and OZSW 2016 (Groningen), for their constructive feedback. The research that led to this paper was generously funded by the Research Foundation Flanders - FWO (Postdoctoral Fellowship).

\section{References}

Bornmann, L., \& Rüdiger, M. (2015). Growth rates of modern science: A bibliometric analysis based on the number of publications and cited references. Journal of the Association for Information Science and Technology, 66, 2215-2222.

Briggman, K. L., \& Bock, D. D. (2012). Volume electron microscopy for neuronal circuit reconstruction. Current Opinion in Neurobiology, 22, 154-161. 
Calcott, B. (2011). Wimsatt and the robustness family: Review of Wimsatt's Reengineering Philosophy for Limited Beings. Biology \& Philosophy, 26, 281-293.

Cartwright, N. (1983). How the laws of physics lie. Oxford: Clarendon Press.

Chakravartty, A. (2007). A metaphysics for scientific realism. Cambridge: Cambridge University Press.

Chang, H. 2004. Inventing temperature: Measurement and scientific progress. Oxford: Oxford University Press.

Clarke, S. (2001). Defensible territory for entity realism. British Journal for the Philosophy of Science, 52, 701-722.

Devitt, M. (2005). Scientific realism. In The Oxford Handbook of Contemporary Philosophy, ed. Frank Jackson and Michael Smith (pp. 767-791). Oxford: Oxford University Press.

Egg, M. (2012). Causal warrant for realism about particle physics. Journal for General Philosophy of Science, 43, 259-280.

Egg, M. (2016). Expanding our grasp: Causal knowledge and the problem of unconceived alternatives. British Journal for the Philosophy of Science, 67, 115-141.

Eronen, M. I. (2012). Pluralistic physicalism and the causal exclusion argument. European Journal for Philosophy of Science 2(2), 219-232. 
Eronen, M. I. (2015). Robustness and reality. Synthese 192(12), 3961-3977.

Fagan, M. B. (2013). The stem cell uncertainty principle. Philosophy of Science 80, $945-$ 957.

Fahrbach, L. (2011). How the growth of science ends theory change. Synthese, 180, 139155.

French, S. (2011). Shifting to structures in physics and biology: A prophylactic for promiscuous realism. Studies in History and Philosophy of Biological and Biomedical Sciences, 42, 164-173.

Frigg, R. \& Votsis, I. (2011). Everything you always wanted to know about structural realism but were afraid to ask. European Journal for Philosophy of Science 1, 227-276.

Guillery, R. W. (2005). Observations of synaptic structures: Origins of the neuron doctrine and its current status. Philosophical Transactions of the Royal Society B, 360 1281-1307. Hacking, I. (1981). Do we see through a microscope? Pacific Philosophical Quarterly, 62, 305-322.

Hacking, I. (1982). Experimentation and scientific realism. Philosophical Topics, 13, $154-$ 172.

Hacking, I. (1983). Representing and intervening. Introductory topics in the philosophy of natural science. New York: Cambridge University Press. 
Hitchcock, C. (1992). Causal explanation and scientific realism. Erkenntnis, 37, 151-178.

Hudson, R. (2014). Seeing things: The philosophy of reliable observation. Oxford: Oxford University Press.

Kolb, H. (2003). How the retina works. American Scientist, 91, 28-35.

Kolb, H. (2015). Roles of amacrine cells. Webvision. Moran Eye Center.

http://webvision.med.utah.edu/book/part-iii-retinal-circuits/roles-of-amacrine-cells/. Accessed 20 February 2017.

Kuorikoski, J., \& Marchionni, C. (2016). Evidential diversity and the triangulation of phenomena. Philosophy of Science, 83, 227-247.

Kuorikoski, J., Lehtinen, A., \& Marchionni, C. (2012). Robustness analysis disclaimer: please read the manual before use! Biology \& Philosophy, 27, 891-902.

Ladyman, J., \& Ross, D. (2007). Every thing must go: Metaphysics naturalised. Oxford: Oxford University Press.

Ladyman, J. (2014). Structural realism. In The Stanford Encyclopedia of Philosophy (Spring 2014 Edition), ed. Edward N. Zalta, http://plato.stanford.edu/archives/spr2014/entries/structural-realism/.

Laudan, L. (1981). A confutation of convergent realism. Philosophy of Science, 48, 19-48. 
Lichtman, J. W., Sanes, J. R., \& Livet, J. (2008). A technicolour approach to the connectome. Nature reviews Neuroscience, 9, 417.

Lyre, H. (2013). Must structural realism cover the special sciences? In EPSA11: Perspectives and Foundational Problems in Philosophy of Science, ed. V. Karakostas \& D. Dieks (pp. 383-390). Dordrecht: Springer.

Masland, R. H. (2012). The tasks of amacrine cells. Visual Neuroscience, 29, 3-9.

Morrison, M. (1990). Theory, intervention and realism. Synthese, 82, 1-22.

O'Conaill, D. (2014). Ontic structural realism and concrete objects. Philosophical Quarterly, 64, 284-300

Odenbaugh, J., \& Alexandrova, A. (2011). Buyer beware: robustness analyses in economics and biology. Biology \& Philosophy, 26, 757-771.

Psillos, S. (1999). Scientific realism: How science tracks truth. London: Routledge.

Radder, H. (2012). The material realization of science. From habermas to experimentation and referential realism (revised edition, with a new postscript). Dordrecht: Springer.

Schupbach, J. (forthcoming). Robustness analysis as explanatory reasoning. British Journal for the Philosophy of Science. 
Soler, L., Trizio, E., Nickles, T., \& Wimsatt, W. C. (eds.) (2012). characterizing the robustness of science: After the practice turn in the philosophy of science. Dordrecht: Springer.

Stegenga, J. (2009). Robustness, discordance, and relevance. Philosophy of Science, 76, $650-661$.

Suárez, M. (2008). Experimental realism reconsidered: How inference to the most likely cause might be sound. In Nancy Cartwright's Philosophy of Science, ed. S. Hartmann, C. Hoefer and L. Bovens (pp. 137-13). New York: Routledge.

van Fraassen, B. (1980). The scientific image. Oxford: Oxford University Press.

van Fraassen, B. (1985). Empiricism in the philosophy of science. In Images of Science, ed. Paul Churchland and Clifford Hooker (pp. 245-308). Chicago: University of Chicago Press.

Weisberg, M. (2006). Robustness analysis. Philosophy of Science, 73, 730-742.

Wimsatt, W. C. (1981). Robustness, reliability, and overdetermination. In Scientific Inquiry and the Social Sciences, ed. M. Brewer and B. Collins (pp. 124-163). San Fransisco: Jossey-Bass.

Wimsatt, W C. (1994). The ontology of complex systems: Levels of organization, perspectives, and causal thickets. Canadian Journal of Philosophy, S20, 207-274. 
Wimsatt, W. C. (2007). Re-engineering philosophy for limited beings. Piecewise approximations to reality. Cambridge, MA: Harvard University.

Zeilinger, A. (2005). The message of the quantum. Nature, 438, 743. 